\title{
Three dimensional geometry characterization using structured light
}

\author{
Paulo J. Tavares ${ }^{*}$, S) \\ University of Porto, Optics and Experimental Mechanics Laboratory, Porto, Portugal. \\ (*) Email: jtavares@fe.up.pt \\ S: miembro de SEDOPTICA / SEDOPTICA member \\ Recibido / Received: 04/08/2012. Revisado / Revised: 07/10/2012. Aceptado / Accepted: 11/10/2012. \\ DOI: $\underline{\text { http://dx.doi.org/10.7149/OPA.45.4.423 }}$
}

\begin{abstract}
:
In this paper I review several studies related with the development of the doctoral thesis of Paulo J. Tavares. The thesis was directed at implementing different three-dimensional surface acquisition methods with structured light fields and essentially describes these implementations, and the difficulties experienced thereof. Several innovative works have been done in the areas of 3D imaging with active image processing, Fourier transform phase analysis, coherent light fringe projection, phase-to-height calibration in phase measurement profilometry, feature detection in planar calibration targets for camera calibration and; fringe distortion correction resourcing to the Optical Transfer Function. This paper presents the main results obtained in those studies which were all performed at LOME - the Optics and Experimental Mechanics Laboratory of the Mechanical Engineering department of Porto University.
\end{abstract}

Key words: Three-dimensional Measurement, Shape Measurement, Optical Metrology, Profilometry, Fringe Projection, Structured Light, Edge and Feature Detection, Tracking, Camera Calibration, Computer Vision.

\section{REFERENCIAS Y ENLACES / REFERENCES AND LINKS}

[1]. P. Tavares, Three Dimensional Geometry Characterization Using Structured Light Fields, PhD Thesis, Department of Mechanical Engineering, Faculty of Engineering of Porto University, Porto (2010).

[2]. M. Takeda, H. Ina, S. Kobayashi, "Fourier-transform method of fringe-pattern analysis for computerbased topography and interferometry", J. Opt. Soc. Am. 72, 156-160 (1982).

[3]. M. Takeda, K. Mutoh, "Fourier-transform profilometry for the automatic measurement of 3-D object shapes", Appl. Opt. 22, 3977-3982 (1983).

[4]. W. W. Macy, "Two-dimensional fringe-pattern analysis", Appl. Opt. 22, 3898-3901 (1983).

[5]. D. J. Bone, H.-A. Bachor, R. J. Sandeman, "Fringe-pattern analysis using a 2D Fourier transform", Appl. Opt. 25, 1653-1660 (1986).

[6]. X. Y. Su, W. J. Chen, "Fourier transform profilometry: A review", Opt. Laser Eng. 35, 263-284 (2001).

[7]. M. Kujawinska, J. Wojciak, "High-accuracy Fourier transform fringe pattern analysis", Opt. Laser Eng. 14, 325-339 (1991).

[8]. K. Creath, "Temporal phase measurement methods", pp. 94-140 in Digital Fringe Pattern Measurement Techniques, D. W. Robinson G. T. Reid, Edts.,Institute of Physics Publishing, Bristol (1993).

[9]. Z. Zhang, D. Zhang, X. Peng, "Performance analysis of a 3D full-field sensor based on fringe projection", Opt. Laser Eng. 42, 341-353 (2004).

[10]. B. A. Rajoub, D. R Burton, M. J. Lalor, "A new phase-to-height model for measuring object shape using collimated projections of structured light", J. Opt. A - Pure Appl. Opt. 7, S368-S375 (2005).

[11].F. Chen, G. Brown, M. Song, "Overview of three-dimensional shape measurement using optical methods", Opt. Eng. 39, 10-22 (2000).

[12]. R. Legarda-Saenz, T. Bothe, W: P. Jüptner, "Accurate procedure for the calibration of a structured light system", Opt. Eng. 43, 464-471 (2004). 
[13]. R. Sitnik, M. Kujawińska, J. Woźnicki, “Digital fringe projection system for large volume 360 deg shape measurement", Opt. Eng. 41, 443-449 (2002).

[14].W.-S. Zhou, X.-Y. Su, "A direct mapping algorithm for phase-measuring profilometry", J. Mod. Opt. 41, 89-94 (1994).

[15]. H. Guo, H. He, Y. Yu, M. Chen, "Least-squares calibration method for fringe projection profilometry", Opt. Eng. 44, 1-9 (2005).

[16].P. Tavares, M. Vaz, "Orthogonal projection technique for resolution enhancement of the Fourier transform fringe analysis method", Opt. Commun. 266, 465-468 (2006).

[17].P. J. Tavares, M. A. Vaz, "Linear calibration procedure for the phase-to-height relationship in phase measurement profilometry", Opt. Commun. 274, 307-314 (2007).

[18]. M. R. Shortis, T. A. Clarke, T. Short, "A comparison of some techniques for the sub-pixel location of discrete target images", Proc. SPIE 2350, 239-250 (1994).

[19]. G. A. W. West, T. A. Clarke, "A survey and examination of sub-pixel measurement techniques", Proc. SPIE 1395, 456-463 (1990).

[20]. C. Schmid, R. Mohr, C. Bauckage, "Evaluation of interest point detectors", Int. J. Comput. Vision 37, 151-172 (2000).

[21]. M. Heath, S. Sarkar, T. Sanocki, K. W. Bowyer, "A robust visual method for assessing the relative performance of edge-detection algorithms", IEEE T. Pattern Anal. 19, 1338-1359 (1997).

[22]. K. Kohlmann, "Corner detection in natural images based on the 2D Hilbert transform", Signal Process. 48, 225-234 (1996).

[23]. H. Stark, "An extension of the Hilbert transform product theorem", Proc. IEEE 59, 1359-1360 (1971).

[24]. T. Bülow, G. Sommer, "Computer analysis of images and patterns", pp. 25-32 in CAIP'99, F. Solina, A. Leonardis, Edts., Ljubljana, Slovenia (1999).

[25].E. Horn, N. Kiryati, "Toward optimal structured light patterns", Proceedings of the 3D DIM 1997 International Conference on Recent Advances of 3D Digital Imaging and Modeling (1997).

[26]. G. Sansoni, M. Carocci, S. Lazzari, R. Rodella, "A three-dimensional imaging system for industrial applications with improved flexibility and robustness", J. Opt. A - Pure Appl. Opt. 1, 83-93 (1998).

[27]. T. Bothe, W. Osten, A. Gesierich, W. Jüptner, “Compact 3D camera”, Proc. SPIE 4778, 48-59 (2002).

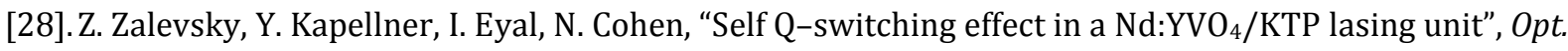
Eng. 45, 070506 (2006).

[29].W. J. Smith, Modern Optical Engineering - The Design of Optical Systems, McGraw Hill, New York (2000).

[30]. R. Guenther, Modern Optics, John Wiley \& Sons, New York (1990).

\section{Introduction}

The work presented herein describes the study of several aspects of three-dimensional geometry characterization using structured light fields. Thus stated, this work has been restricted to a small domain of active image processing, wherefore some sort of interaction occurs between the acquisition apparatus and the scene itself. This interaction, usually in the form of an energy exchange - such as can be done by shedding light onto the surface under study and collecting it with an imaging device - marks the departure of these methods from the more popular passive imaging ones, which make up for the absence of such interaction with the use of two or more imaging devices. Still, and even if these are very different methodologies, there are a number of similarities and contact areas between the two domains. Such is the case with calibration, for instance, which runs along the same lines whatever the chosen image processing method may be.

When choosing the most appropriate method to face a certain situation, several factors must be carefully weighted, such as whether it is a static or quasi-static situation, if there's a controlled lighting environment such as can be found in a laboratory, or if it is a simple or a rather complex geometry. It is not uncommon to find the development of active image processing 
methods in connection to other imaging techniques in experimental mechanics research groups. Actually, this work has been devised in such a way as to bridge the gap between the non destructive testing techniques, which constitute the core of our laboratory work, and surface measurement and characterization, which now becomes available in the sequence of this research.

The original contributions worth mentioning from this work [1] were:

- A method for increasing the resolution of Fourier Transform phase analysis at the expense of acquiring one further image, whenever this can be indulged. The second image is acquired with the grating projected at a right angle to the first one, in order to fully eliminate the central DC frequency in reciprocal space and increase the filter width twice;

- A novel approach to phase-to-height calibration, which uses just two images, significantly reducing the usual image acquisition and processing burden for this absolutely indispensable calibration step;

- A sub-pixel corner detection algorithm for camera calibration. Separation of the intrinsic and extrinsic calibration parameters detection was implemented in a very flexible way, to facilitate camera repositioning;

- A coherent light projection system was further developed and tested under broad day light, which seems to be a very promising technique, enabling the use of active image processing outside of carefully controlled lighting environments, and;

- A simple method to correct fringe distortion, which relies on the calculation of the projection acquisition system's optical transfer function.

\section{Orthogonal projection technique in Fourier transform fringe analysis method}

\section{2.a. Introduction}

The Fourier transform fringe analysis method is simple and effective, yet plagued with many error sources [1-6]. One of the most important is leakage - the error that results from the fact that the frequencies spread out to infinity in frequency space, and a too wide filtering window is bound to include frequencies that belong to adjacent orders and therefore "leak" into the observed window. This is a stringent constraint, in that the filter size is very limited and a number of high frequencies responsible for the image detail can thus be lost. What we propose herein, is a simple technique to eliminate the zero order in frequency space and widen the filter window size to approximately twice the maximum admissible size without leakage from the adjacent orders. The resolution enhancement so obtained is double the resolution from the original method.

\section{2.b. Analysis}

A sinusoidal fringe pattern projected onto an object is adequately described by,

$$
\begin{aligned}
g_{x}(x, y)= & a(x, y)+ \\
& +b(x, y)\left[\cos \left(2 \pi f_{0} x+\phi(x, y)\right)\right],
\end{aligned}
$$

where $a(x, y)$ and $b(x, y)$ represent nonuniform distributions of reflectivity on the surface of the object, $f_{0}$ is the fundamental frequency of the observed grating image and $\phi(x, y)$ is the phase modulation resulting from the object height distribution. For convenience of analysis, this fringe pattern can be rewritten as,

$$
\begin{aligned}
g_{x}(x, y)=a(x, y) & +c(x, y) \exp \left(j 2 \pi f_{0} x\right)+ \\
& +c^{*}(x, y) \exp \left(-j 2 \pi f_{0} x\right),
\end{aligned}
$$

with the understanding that,

$$
c(x, y)=\frac{1}{2} b(x, y) \exp (j \phi(x, y)),
$$

and "*" denotes complex conjugation. A pattern projected at a right angle to the first can therefore be written as, 


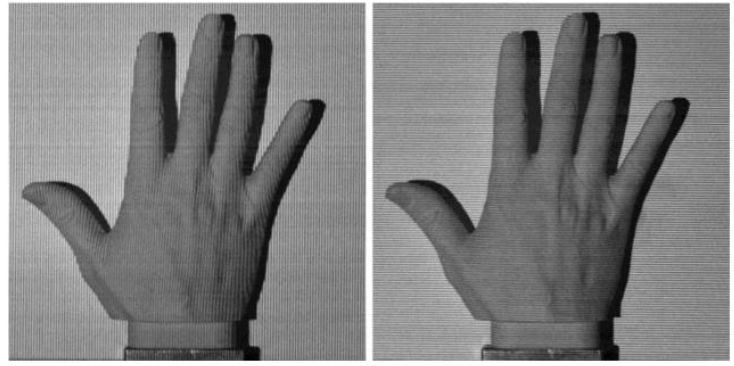

Fig. 1. Projected fringe patterns.

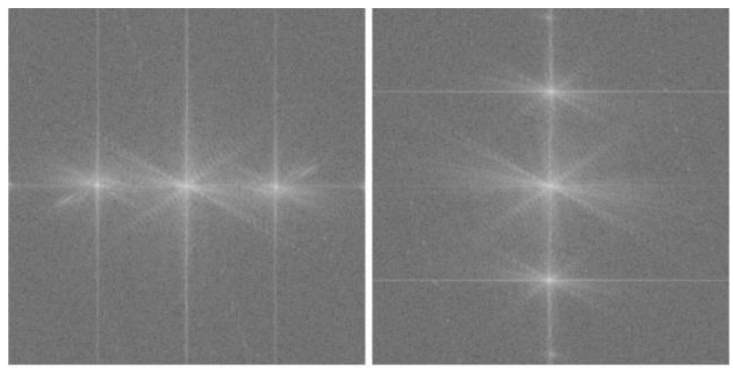

Fig. 2.Fourier spectra of orthogonal patterns.

$$
\begin{aligned}
g_{y}(x, y)=a(x, y) & +c(x, y) \exp \left(j 2 \pi f_{0} y\right)+ \\
& +c^{*}(x, y) \exp \left(-j 2 \pi f_{0} y\right) .
\end{aligned}
$$

Two such sinusoidal orthogonal patterns can be observed in Fig. 1.

The Fourier transforms of the deformed fringe patterns, shown in Fig. 2, are,

$$
\begin{aligned}
G_{u}(u, v)= & A(u, v)+C\left(u-f_{0}, v\right)+ \\
& +C^{*}\left(u+f_{0}, v\right),
\end{aligned}
$$

and

$$
\begin{aligned}
G_{v}(u, v)= & A(u, v)+C\left(u, v-f_{0}\right)+ \\
& +C^{*}\left(u, v+f_{0}\right) .
\end{aligned}
$$

It is clear from the transform equations above and the examination of both spectra that the central dc term is equal in both instances, in case the contrast and gain of the two fringe patterns is kept constant and the background intensity variation under control, which suggest a simple form to remove it. Subtraction of Eqs. (5) and (6) above produces,

$$
\begin{aligned}
G_{u}(u, v) & -G_{v}(u, v)= \\
= & C\left(u-f_{0}, v\right)-C\left(u, v-f_{0}\right)+ \\
& +C^{*}\left(u+f_{0}, v\right)-C^{*}\left(u, v+f_{0}\right),
\end{aligned}
$$

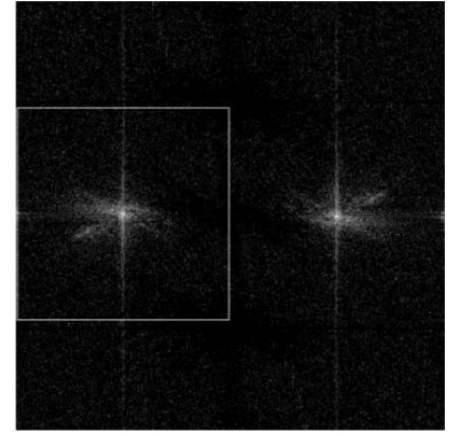

Fig. 3. Retained frequencies.
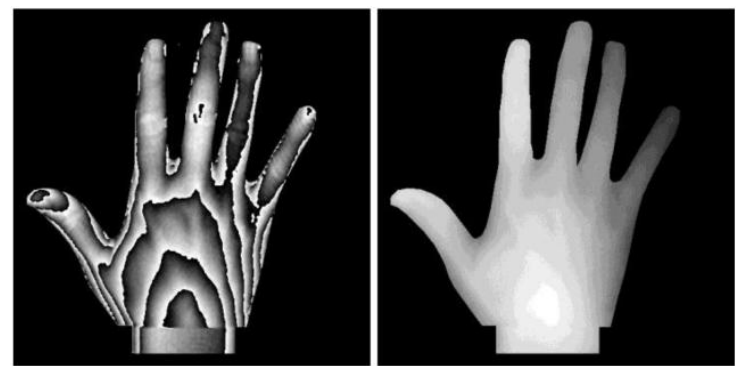

Fig. 4. Obtained phase.

which means that keeping just the positive part of this equation, i.e., dropping the negative part of the resulting image altogether, results in

$$
C\left(u-f_{0}, v\right)+C^{*}\left(u+f_{0}, v\right) .
$$

It is interesting to note that any visibility variation between the two original images will be totally eliminated by this clipping operation. The resulting spectrum can be seen in Fig. 3, together with the mask limits that can now be used for carrier removal, which span an area twice as large as the original filter's. The outcome of this mask enlargement is a higher resolution phase map that can be seen in Fig. 4 together with the unwrapped 3D point cloud result.

\section{2.c. Conclusions}

The Fourier transform profilometry method for 3D surface measurement has become very popular due to the fact that it is inherently simple, standing out as one of the very few methods applicable to dynamic situations because it relies on a single image frame. One of the method's chief restrictions is the limited resolution, due to the well-known leakage artifact from the application of FFTs and the constraint it imposes on the carrier removal filter size. 
The method presented in this note removes this limitation to a great extent, by eliminating the central DC frequency component in frequency space, thus allowing the filter's size to be twice the carrier frequency.

\section{Linear phase-to-height calibration in Fourier transform fringe analysis}

\section{3.a. Introduction}

Camera calibration is but one part of the calibration procedure of a phase measurement instrument. Phase measurement techniques [17] calculate phase-shift mappings of surface deformations or profile-to-reference differences and rely, at some point, on the translation of the obtained phase map to either a dislocation or a height measurement. The relation between phase and height has thus been the subject of a large number of works over the years, due to its impact on the overall method's accuracy. These works can be divided in analytical $[3,6,9,10]$ and empirical approaches [12-14].

\section{3.b. Analysis}

Figure 5 depicts a typical optical crossed axis fringe projection setup for phase measurement. A fringe pattern with a known spatial frequency is projected onto the object under test by projector $P$ and the image is captured at camera $C$. $E_{P}$ and $E_{C}$ refer to the nodal points of the projection and imaging systems, respectively. The optical axes of these systems cross at point $O$ on a reference plane $R_{0} . L_{0}$ is the distance from the camera-projector plane to the reference plane, $d$ is the distance between centres of projection, $q$ is the angle between projector and camera and $s$ represents the intersection of $E_{P} B$ with the object surface. $E_{T}$ represents a telecentric projection through s, described by,

$$
g_{T}(x, y)=a(x, y)+b(x, y) \cos \left(2 \pi f_{0} x\right),
$$

where $a(x, y)$ and $b(x, y) / a(x, y)$ represent, as before, the average intensity and fringe visibility or modulation on the surface of the object, $f_{0}$ is the fundamental frequency of the observed grating image, and the fringes run perpendicular to the paper plane. In the non-telecentric case, the pattern on the reference plane can be described by,

$$
\begin{aligned}
& g_{o}(x, y)=a(x, y)+ \\
& \quad+b(x, y) \cos \left(2 \pi f_{0} x+\phi_{0}(x, y)\right),
\end{aligned}
$$

with

$$
\phi_{0}(x, y)=2 \pi f_{0} \overline{B D} .
$$

When the object is in place, the ray from $E_{P}$ that strikes the reference plane at $B$ is seen from the camera as coming from $A$, and the phase expression for $h(x, y) \neq 0$ is therefore

$$
\phi(x, y)=2 \pi f_{0} \overline{A D} .
$$

The fringe pattern on the object surface is expressed by

$$
\begin{aligned}
& g_{S}(x, y)=a(x, y)+ \\
& \quad+b(x, y) \cos \left(2 \pi f_{0} x+\phi(x, y)\right),
\end{aligned}
$$

and the phase difference between reference plane and object surface is

$$
\Delta \phi(x, y)=2 \pi f_{0}(\overline{A D}-\overline{B D})=2 \pi f_{0} \overline{A B} .
$$

As shown by Takeda et al [2], this phase difference is completely recovered as,

$$
\Delta \phi(x, y)=\operatorname{Im}\left\{\log \left[G_{S}(u, v) G_{O}^{*}(u, v)\right]\right\} .
$$

where $G_{S}(u, v)$ is the Fourier Transform of the fringe pattern and * denotes complex conjugation. In order to establish a relation between phase and height, one uses the fact that triangles $E_{P} S E_{C}$ and $A_{S} B$ are similar

$$
\overline{A B}=\frac{d h}{L_{0}-h},
$$

so

$$
\begin{aligned}
& \Delta \phi(x, y)=2 \pi f_{0} A B \Rightarrow \\
& \Rightarrow h(x, y)=\frac{L_{0} \Delta \phi(x, y)}{\Delta \phi(x, y)+2 \pi f_{0} d} .
\end{aligned}
$$

A thorough analysis in [8] indicates $h(x, y)$ is in fact a function of the lateral coordinate $x$ and the angle between projector and camera as,

$$
h(x, y)=\frac{L_{0}}{\frac{2 \pi L_{0}^{2} d \cos \theta}{P_{0} \Delta \phi(x, y)\left(L_{0}+x \cos \theta \sin \theta\right)^{2}}-\frac{d \cos \theta \sin \theta}{L_{0}+x \cos \theta \sin \theta}+1} .
$$




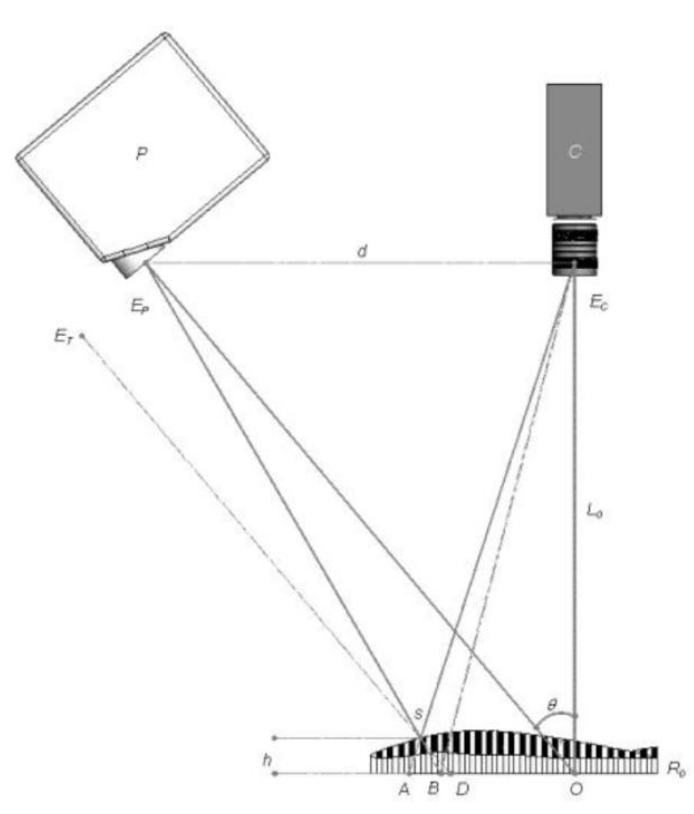

Fig. 5. Typical structured light phase measurement setup.

with

$$
P_{0}=\frac{1}{f_{0}}=\frac{P}{\cos \theta},
$$

$P$, being the projected grating period.

Now, an analysis of either expression for $h(x, y)$ will reveal they are not that much different for a plausible application regime. Reversing the last function for $h(x, y)$ above gives

$$
\Delta \phi=\frac{2 h \pi L_{0}^{2} d \cos \theta}{P_{0}\left(h A+L_{0}^{3}+B\right)}
$$

with

$$
A=d L_{0} \cos \theta \sin \theta+d x \cos ^{2} \theta \sin ^{2} \theta-L_{0}^{2},
$$

And

$$
\begin{aligned}
& B=\left(L_{0}-h\right) x \cos \theta \sin \theta \times \\
& \times\left(2 L_{0}+x \cos \theta \sin \theta\right),
\end{aligned}
$$

Figure 6 shows a comparison of the former expressions for $\Delta \phi(x, y)$ taken as functions of $h(x, y)$ in both cases. Several plots of Eq. (20) corresponding to different values of $x$ ranging from 0 to $100 \mathrm{~mm}$ are present, even though any of these would suffice to describe the function correctly.

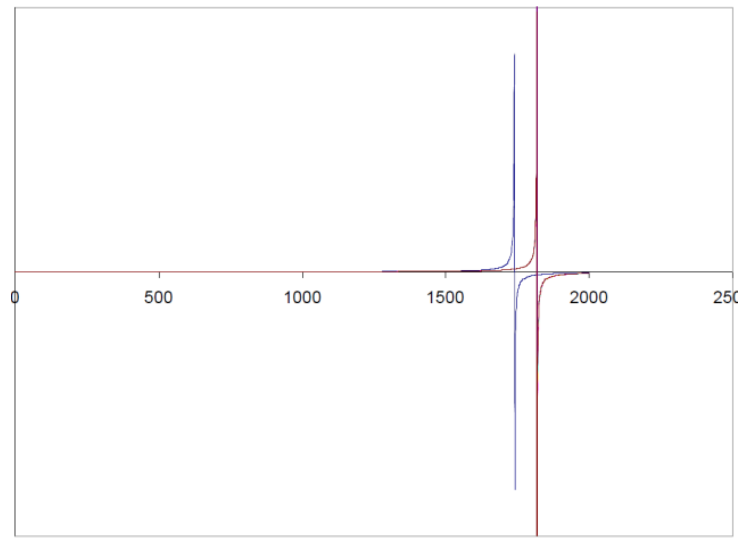

Fig. 6. The plot of $\Delta \phi(x, y)$ with varying $h(x, y)(\mathrm{mm})$.

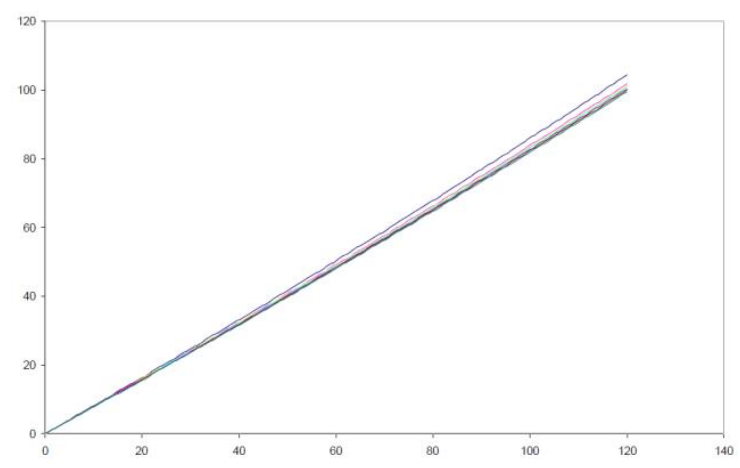

Fig. 7. The plot of $\Delta \phi(x, y)$ with varying $h(x, y)$. Detail for 0 $120 \mathrm{~mm}$.

This plot was done for $L_{0}=1740 \mathrm{~mm}, d=370$ $\mathrm{mm}, f_{0}=0.6$ fringes $/ \mathrm{mm}$ and a field size of 160 $\mathrm{mm}$. The plots are nearly identical except for the region around camera location.

The highly non-linear discontinuity regions occur near or past the camera location and are totally out of reach for ordinary phase measuring profilometry techniques, because of both depth of focus and the steepness limit of data acquisition. Even with the improved data range described in [5], the maximum observable height for the $160 \mathrm{~mm}$ plane would be approximately $78 \mathrm{~mm}$. If one compares the region of 0 to $100 \mathrm{~mm}$ for instance the plots are very similar - cf. Fig. 7.

Moreover, the analytical plots are strikingly linear at these regimes. The correlation coefficient for $h$ in Eq. (17) and the first $120 \mathrm{~mm}$ is $R^{2}=0.9997$ on this plot.

Experimental methods [12-14] readily corroborate these findings for measurement ranges that stay within a useful region and 
therefore one can safely calibrate the phase-toheight relation with a linear calibration procedure. Higher order polynomial interpolation will not improve the method significantly.

In view of this fact, a simple, yet effective, linear calibration procedure has been devised in LOME. Although the procedure described herein requires a priori accurate camera calibration and the possibility to calibrate for rigid body motion within the depth of field, it has the advantage of requiring just one phase map of an inclined reference plane and can therefore accelerate the complete process of calibrating phase-to-height.

A necessary step for camera calibration is the acquisition of the image location of a set of points, traditionally on some sort of calibration pattern. The result from the extrinsic camera calibration is a set of data describing a rigid body transformation. Basically it transforms the World Coordinate System $\left(X_{w}, Y_{w}, Z_{w}\right)$ (WCS1) into a Camera Coordinate System $\left(X_{c}, Y_{c}, Z_{c}\right)$ (CCS) as depicted in Fig. 8. If we now rotate the calibration plane to a new position and recalibrate the camera, we will obtain a new transformation, this time from $\left(X_{w 2}, Y_{w 2}, Z_{w 2}\right)$ to $\left(X_{c}, Y_{c}, Z_{c}\right)$ (WCS2).

We describe the transformations on projective space with homogeneous coordinates, so the complete transformation, consisting of one rotation followed by one translation is reduced to just one operation

$$
[\operatorname{Tr}]=[R][T],
$$

with

$$
[R]=\left[\begin{array}{cccc}
R_{00} & R_{01} & R_{02} & 0 \\
R_{10} & R_{11} & R_{12} & 0 \\
R_{20} & R_{21} & R_{22} & 0 \\
0 & 0 & 0 & 1
\end{array}\right]
$$

and

$[\operatorname{Tr}]=$

$=\left[\begin{array}{cccc}R_{00} & R_{01} & R_{02} & R_{00} T_{x}+R_{01} T_{y}+R_{02} T_{z} \\ R_{10} & R_{11} & R_{12} & R_{10} T_{x}+R_{11} T_{y}+R_{12} T_{z} \\ R_{20} & R_{21} & R_{22} & R_{20} T_{x}+R_{21} T_{y}+R_{22} T_{z} \\ 0 & 0 & 0 & 1\end{array}\right]$

If $\left[\mathrm{Tr}_{1}\right]$ describes the transformation from WCS1 to CCS and $\left[\mathrm{Tr}_{2}\right]$ the transformation from WCS2 to CCS, the transformation we seek, describing points of WCS2 in WCS1, will be given by

$$
\begin{aligned}
{\left[\begin{array}{c}
X_{w 1 i} \\
Y_{w 1 i} \\
Z_{w 1 i} \\
1
\end{array}\right]=[\operatorname{Tr}]\left[\begin{array}{c}
X_{w 2 i} \\
Y_{w 2 i} \\
Z_{w 2 i} \\
1
\end{array}\right]=} \\
=\left[\operatorname{Tr}_{1}\right]^{-1}\left[\operatorname{Tr}_{2}\right]\left[\begin{array}{c}
X_{w 2 i} \\
Y_{w 2 i} \\
Z_{w 2 i} \\
1
\end{array}\right],
\end{aligned}
$$

The transformation above calculates the position of the rotated calibration points on WCS1.

A phase map is calculated on each position and subtracted to obtain a phase difference map between both positions. If we define $z=0$ on WCS1 we will have a set of points with different zi coordinates and to each of which we have a corresponding phase difference, $\Delta \phi_{i}(x, y)$. A simple least squares fitting of $z_{i}$ coordinates to phase differences will result on a couple of global coefficients $a$ and $b$ such that,

$$
z_{i}(x, y)=a+b \Delta \phi(x, y)
$$

We have tested this procedure against a least squares $z$-shift described in [15] on a plaster mold shown in Fig. 9.

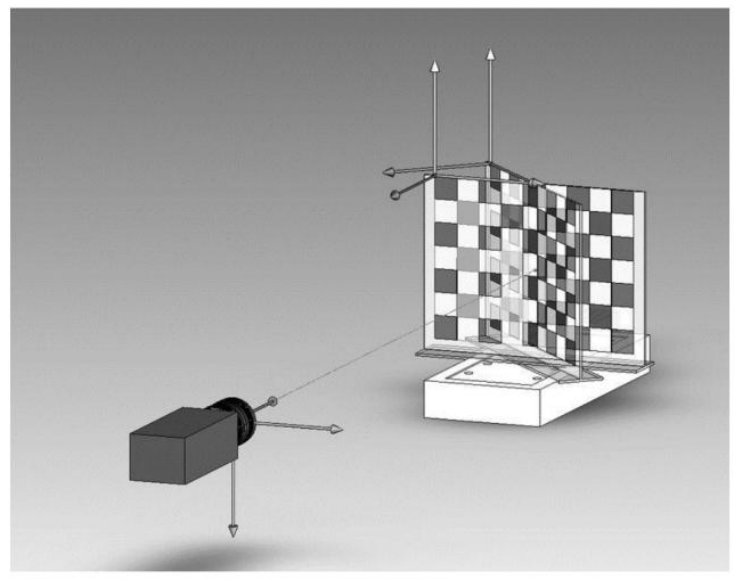

Fig. 8. One frame phase-to-height calibration procedure coordinate systems.

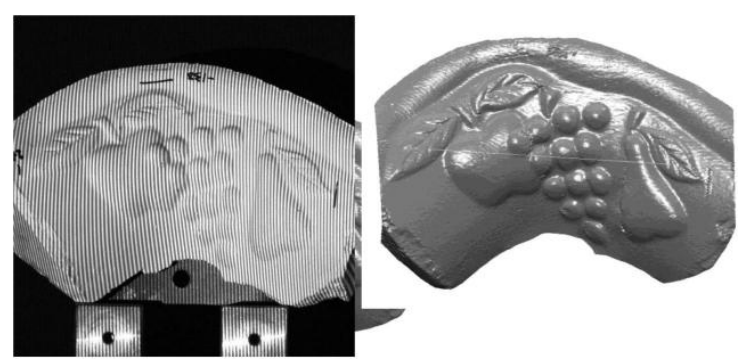

Fig. 9. Plaster mold used in testing and tessellated model after profilometry completion. 


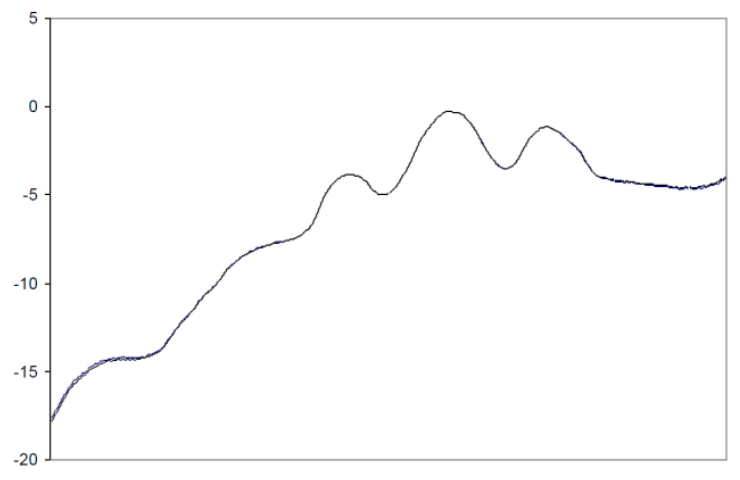

Fig. 10. Column\# 255 on the 3D point cloud of the plaster model 3.

This part, though most of its relief is quite simple to handle, has both a very steep border and a high surface curvature. The part is $70 \mathrm{~mm}$ thick and $55 \mathrm{~mm}$ high. The lateral dimension recovered was $160 \mathrm{~mm}$. We calibrated the $z$-shift phase-to-height relation using a full 100, $1 \mathrm{~mm}$ shift interpolation reference plans and the proposed technique with the reference plan at 0 and 42 degrees.

The final surface was compared against a high resolution 3D model of the part made by contact profiling. The differences, both in height and in XY coordinates, between the 3D models seem to indicate very small errors, between 0.01 and $0.1 \mathrm{~mm}$, though the exact positioning of the measurement markers on the 3D models may not be as accurate.

A profile of column 255 on the 3D point cloud recovered through both the least squares $z$-shift and our own procedure can be seen in Fig. 10. Small deviations between both models can be observed at the beginning and end of the lines, which we think may be attributed to the camera calibration method employed.

\section{3.c. Conclusions}

Phase measurement profilometry methods are amongst the most popular non-contact 3D surface measurement techniques. These are active image processing methods which thus need calibration of the instrument-object interfering mechanism. With PMP methods this requirement translates into a calibration of the relation between phase and object height, which is traditionally obtained by shifting a reference plane perpendicularly to the camera, obtaining phase maps at each shifting step and relating phase-to-height by fitting some sort of function to the calculated phase maps. This is an awkward and lengthy process, which has to be performed in controlled conditions and adequate equipment inside a laboratory.

The method we have proposed, though it requires accurate camera calibration, reduces this process to the acquisition of just one further phase map of the reference plane at an inclined position. Camera calibration data combined with the obtained phase maps provides sufficient information to completely describe the relation between phase and height. We have shown this is feasible and simple, and the results are sufficiently accurate.

\section{A sub-pixel corner detector for camera calibration}

Feature detectors have long been one of the touchstones of image processing. Most vision tasks are entirely dependent on the accurate determination of fiducial marks on images, which ultimately led to a quest for methods able to detect feature locations with high resolution. This note reports the development of an intensity based sub-pixel corner detector based on the 2D Hilbert transform. The method uses a planner checkerboard calibration target and detects double corner locations using a bidimensional Hilbert transform, a method based on Kohlmann's proposal. The Hilbert transform results in a peak at each corner location and a sub-pixel algorithm is then applied at these positions. Both a squared centroid and a ridge peak detection by paraboloid fitting were used with no appreciable differences. Extensive testing of both, accuracy and precision with live images, ascertain the method as adequate for sub-pixel detection at better than $10^{-1}$ pixel accuracy.

\section{4.b. Analysis}

The Hilbert transform has been used for a long time in communications theory and frequency analysis wherefore it provides a practical means of isolating the frequency components of a given function. Its application to corner detection is thoroughly described in [22] and [23]. A successful extension of $1 \mathrm{D}$ to $2 \mathrm{D}$ analytical 
signals based on the Hilbert Transform is described in [24].

Let $s\left(t_{1}, t_{2}\right)$ be a real integrable function. Its Fourier transform is given by

$$
\begin{aligned}
& S\left(\omega_{1}, \omega_{2}\right)= \\
& \quad=\iint_{-\infty}^{+\infty} s\left(t_{1}, t_{2}\right) e^{-j \omega_{1} t_{1}} e^{-j \omega_{2} t_{2}} d t_{1} d t_{2} .
\end{aligned}
$$

and it's Hilbert transform by

$$
\begin{array}{r}
\hat{s}\left(t_{1}, t_{2}\right)= \\
=\frac{p \cdot v \cdot}{\pi^{2}} \int_{-\infty}^{+\infty} \frac{s\left(\tau_{1}, \tau_{2}\right)}{\left(t_{1}-\tau_{1}\right)\left(t_{2}-\tau_{2}\right)} d \tau_{1} d \tau_{2}= \\
=\frac{1}{\pi^{2} t_{1} t_{2}} \otimes s\left(t_{1}, t_{2}\right),
\end{array}
$$

where $p . v$. refers to the principal value of the integral and $\otimes$ is the convolution operator. The Fourier transform of $1 /\left(\pi^{2} t_{1} t_{2}\right)$ is

$$
\begin{aligned}
& F\left(\frac{1}{\pi^{2} t_{1} t_{2}}\right)= \\
& =-j\left[1-2 H\left(-\omega_{1}\right)\right]-j\left[1-2 H\left(-\omega_{2}\right)\right]= \\
& =-\operatorname{sgn}\left(\omega_{1}\right) \operatorname{sgn}\left(\omega_{2}\right),
\end{aligned}
$$

where $H(x)$ is the Heavyside function and $\operatorname{sgn}(x)$ is the signum function.

By the convolution theorem, the Fourier transform of the Hilbert transform of $s\left(t_{1}, t_{2}\right)$ relates to the Fourier transform of $s\left(t_{1}, t_{2}\right)$ by

$$
\begin{aligned}
& \hat{S}\left(\omega_{1}, \omega_{2}\right) \equiv F\left(\hat{s}\left(t_{1}, t_{2}\right)\right)= \\
& =-\operatorname{sgn}\left(\omega_{1}\right) \operatorname{sgn}\left(\omega_{2}\right) F\left(\mathrm{~s}\left(t_{1}, t_{2}\right)\right) \equiv \\
& \equiv-\operatorname{sgn}\left(\omega_{1}\right) \operatorname{sgn}\left(\omega_{2}\right) S\left(\omega_{1}, \omega_{2}\right) .
\end{aligned}
$$

For a Linear Shift Invariant (LSI) system with impulse response $1 /\left(\pi^{2} t_{1} t_{2}\right)$, the function $-\operatorname{sgn}\left(\omega_{1}\right) \operatorname{sgn}\left(\omega_{2}\right)$ represents the corresponding frequency response and serves as a Hilbert transformator. This system responds to steep changes in image frequency, such as those in edges, in a way very similar to spatial differentiation, except for the fact that it is more stable to frequency oscillations.

If the calibration pattern is composed of squares, such as the one in Fig. 11, the Hilbert transform can be thought of as a separable function in a two dimensional space. When applied along direction $x x$, it detects the edges in that direction, as shown in Fig. 12.

If it is now applied along the $y y$ direction to the result of the first transformation, it will detect the edges along the new direction, i.e., the corners of the given squares. This is the basis of the method, wherefore the absolute values are retained after calculating the transforms, cf. Fig. 13.

Regions of interest are established around each peak through a Gaussian filter, which reduces most of the unwanted peaks and subsequent binarization of the resulting image, to retain just the central, stronger peak regions. Finally, a peak detection algorithm is applied to the regions of interest in the original peaks image, centered at the intensity maxima of subregions around each binary region, to find exact sub-pixel locations of the respective corner. Two peak detection methods were compared on this study: centroiding and ridge detection by paraboloid fitting.

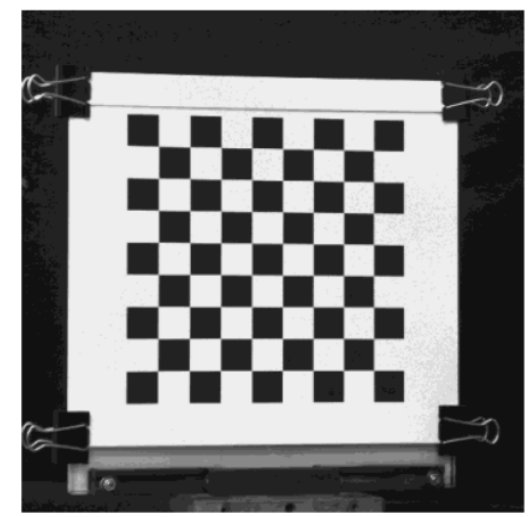

Fig. 11. Camera calibration pattern.

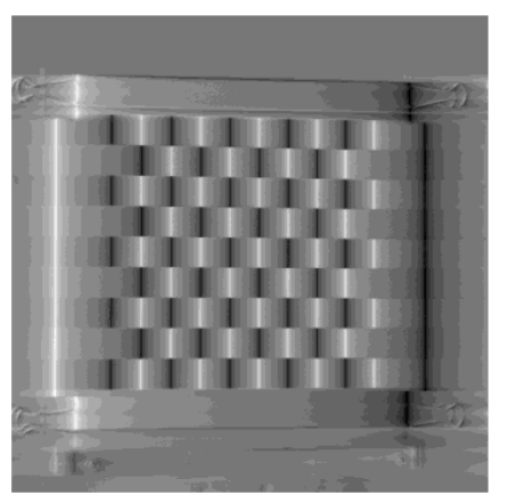

Fig. 12. Hilbert transform along $x x$. 

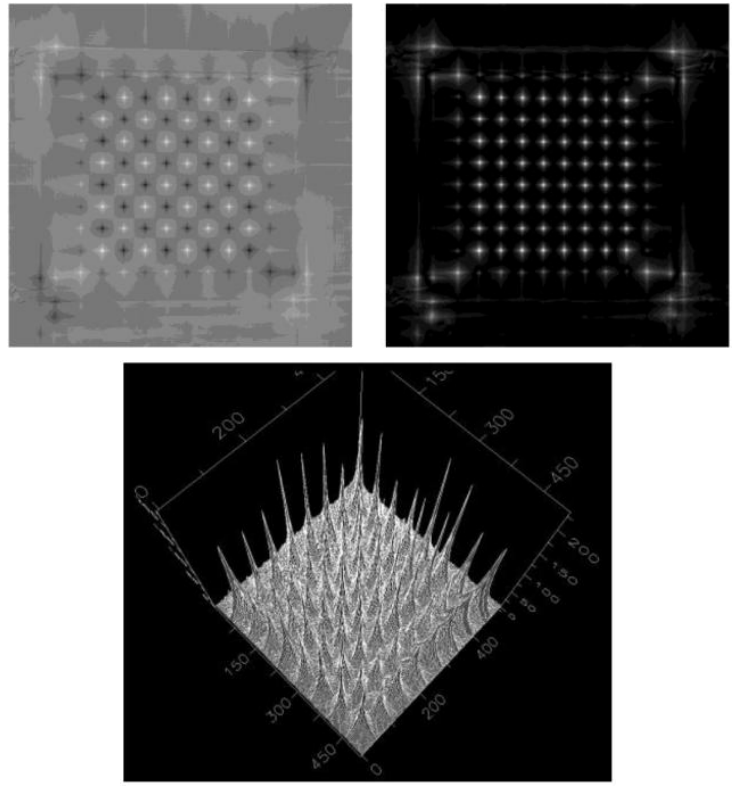

Fig. 13. Hilbert transform along $x x$ and $y y$ and its absolute value.

The method's accuracy was established at or beyond 0.1 pixels, and extensive testing has shown maximum standard deviation below 0.09 pixels. 3-D reconstruction accuracy was calculated, performing camera calibration with the acquired corner positions, which were then projected back through the camera calibration matrix to determine the world coordinates of the observed corners. The distance between these coordinates and the given target locations was calculated for each corner and the ratio of these distances to the image scene width was established at or below $1 / 2000$, which is a fair estimate of $3 \mathrm{D}$ reconstruction accuracy.

\section{Development and implementation of a coherent fringe projection system}

\section{5.a. Introduction}

Active image processing full field methods for 3D contactless profilometry are amongst the current methods of choice for obtaining point clouds from object surfaces. The fringe projection system plays a decisive role on the entire process, significantly impacting both quality and reliability of the final measurements. Moreover, most every phase measurement profilometer can only be used under laboratory controlled lighting environments. This note describes the successful implementation of a coherent fringe projection system in LOME, which enables outdoor measurements by selectively band pass filtering the projected wavelength.

\section{5.b. Analysis}

The laser source used in this work is a $60 \mathrm{~mW}$, $532 \mathrm{~nm}$ DPSS laser source which was developed for this project. The source was designed with an output beam divergence of $30^{\circ}$ in order to fill the useful area of a 2 inch condenser group at 86 $\mathrm{mm}$. It consists on a $2 \mathrm{~W}, 808 \mathrm{~nm} \mathrm{C}$-Mount diode laser pump, a Diode Pumped Microchip (DPM) laser crystal combining a $\mathrm{Nd}: \mathrm{YvO}_{4}$ crystal and a KTP frequency doubler, and a GRIN lens. The DPM1102 module from CASIX is in fact an optically bonded hybrid crystal, which endures much higher powers than previous optically cemented KTP to $\mathrm{Nd}: \mathrm{YvO}_{4}$ crystals. According to CASIX, optical bonding is based on diffusion. The crystals are permanently bonded by heating to a high temperature under pressure resulting in a chemical exchange of molecules at the interface. Even so, there seems to be an ongoing discussion about the properties of these DPM modules, as can be seen in [28].

In order to achieve alignment of the C-Mount pumping diode and the DPM crystal, an XYZ micropositioner was modified to accommodate tilt, as can be seen in Fig. 15. Accurate positioning is very important due to the fact these laser modules have a rather high sweet spot.

The green, $532 \mathrm{~nm}$, beam has a very small divergence at the KTP output side. In order to fill a 2 inch condenser group at a reasonably small distance, the required beam divergence was calculated at $30^{\circ}$, which in turn involved using a very short focal distance focusing lens. We have used a PCH022 GRIN lens from CASIX with a $1 \mathrm{~mm}$ focal distance at $45 \mathrm{~mm}$ from the KTP exit as shown in Fig. 16.

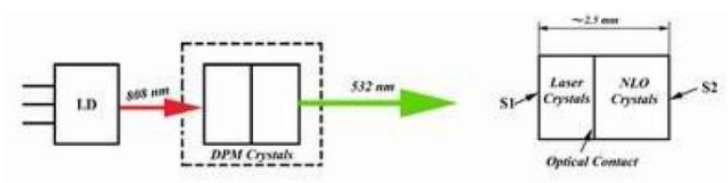

Fig. 14. High power DPM laser modules. 


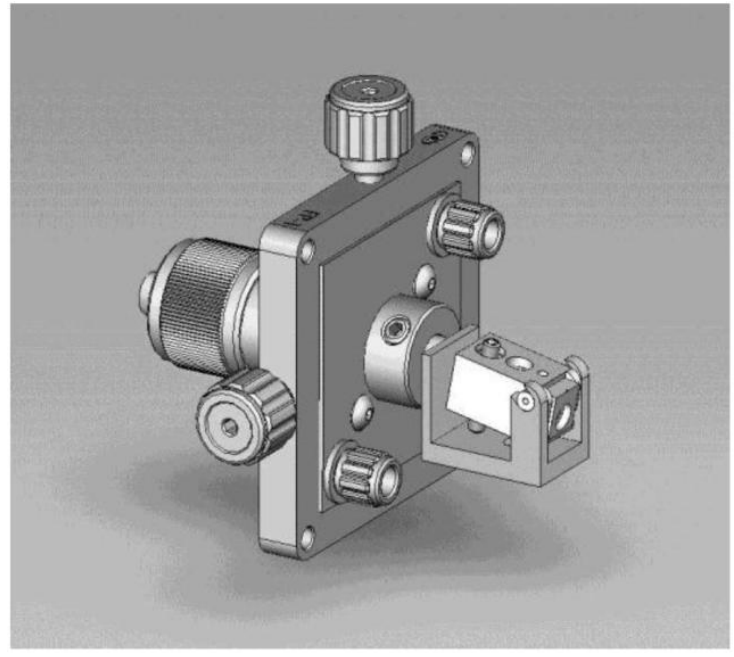

Fig. 15. C-mount laser diode positioning.

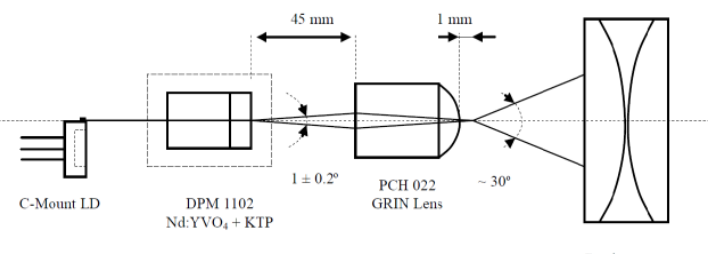

Fig. 16 Beam forming schematics.

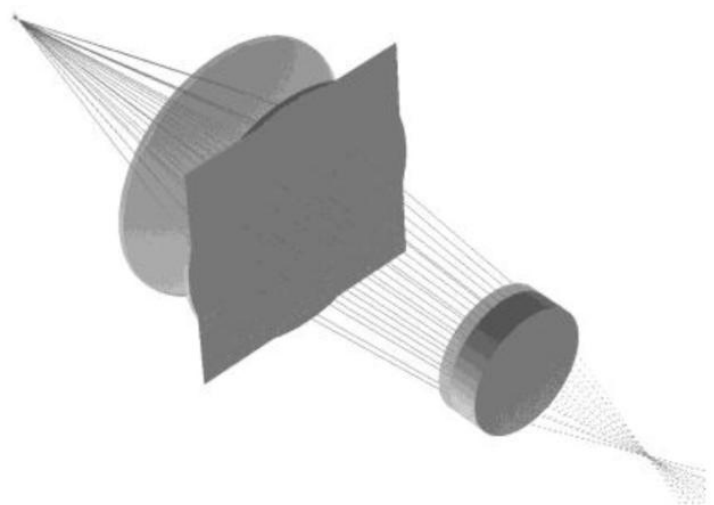

Fig. 17. Ray tracing study of the condenser and projection groups.

The condenser group was calculated having in mind a versatile projection group such as an F-Mount Nikon objective with a typical $46.5 \mathrm{~mm}$ back focal distance. Ray tracing the entire optics assembly, from the GRIN lens forward, is shown in Fig. 17. The condenser group comprises two $50 \mathrm{~mm}$ diameter plano-convex lenses with an 80 $\mathrm{mm}$ focal distance, convex sides apposed, for a better control of spherical aberration.

The spatial light modulator (SLM) used herein was a XGA1 twisted nematic LCD from
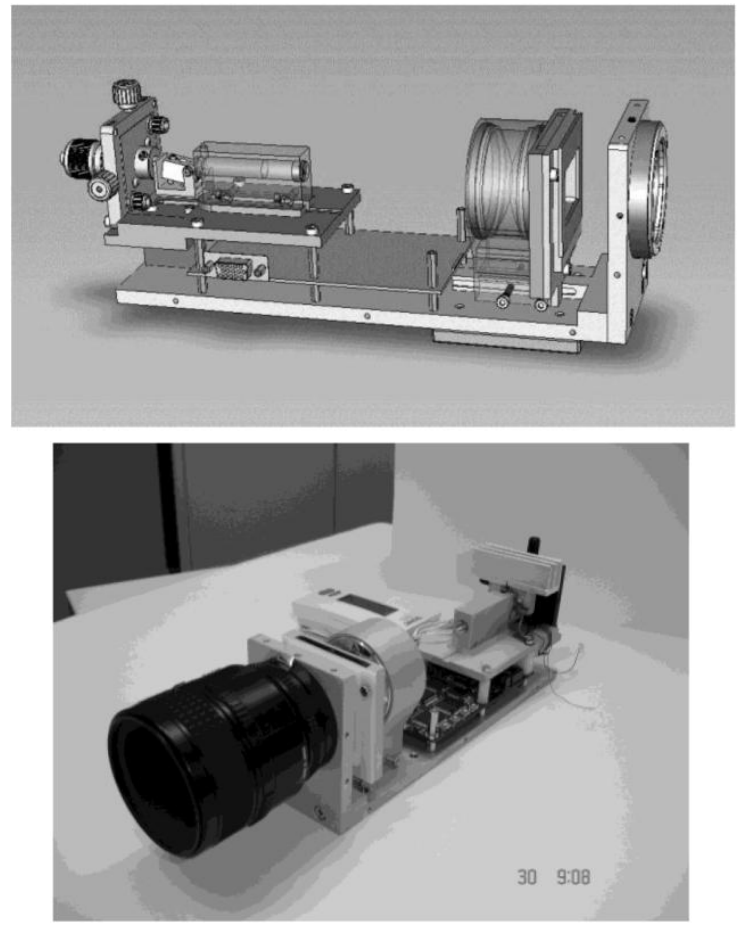

Fig. 18. Complete assembly.

CRL with a $1024 \times 768$ VGA resolution. The entire assembly project can be seen in Fig. 18. In case a higher resolution is necessary, the LCD can be rapidly exchanged with a Ronchi ruling

\section{5.c. Testing}

Testing was performed with both temporal phase unwrapping, which is basically a phaseshift method, and Fourier transform profilometry, which can be seen in Figs. 19 and 20.

\section{5.d. Conclusions}

Active image processing techniques for 3D shape measurement rely heavily on the quality of the fringe projecting system. An innovative compact design for such a system using a coherent light source was projected and assembled in LOME, and its preliminary version was presented in this note.

The device enables the use of phase measurement techniques by fringe projection outside of laboratory controlled lighting. Several problems remain to be solved as the project is yet to be completed. The preliminary test results compare very favorably to other illumination sources, although a complete characterization remains to be done. 

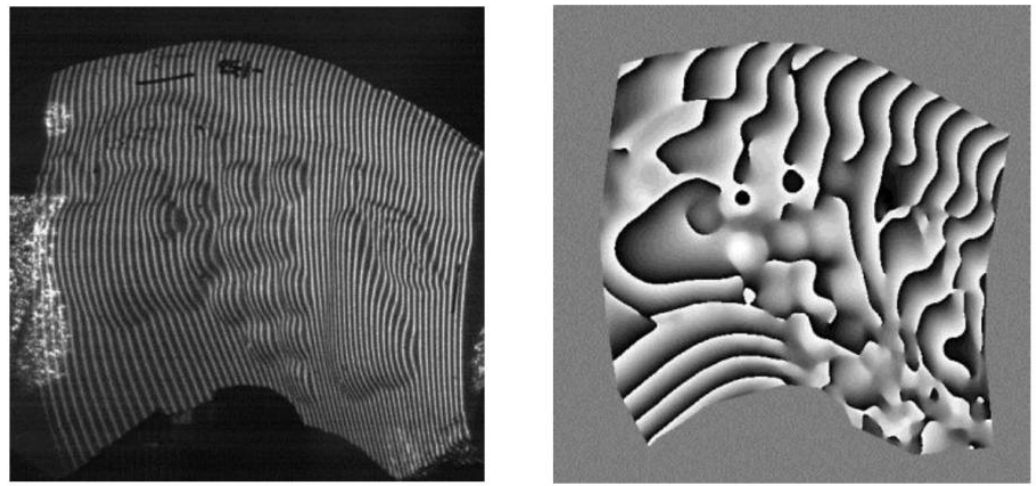

Fig. 19. Plaster mould testing.
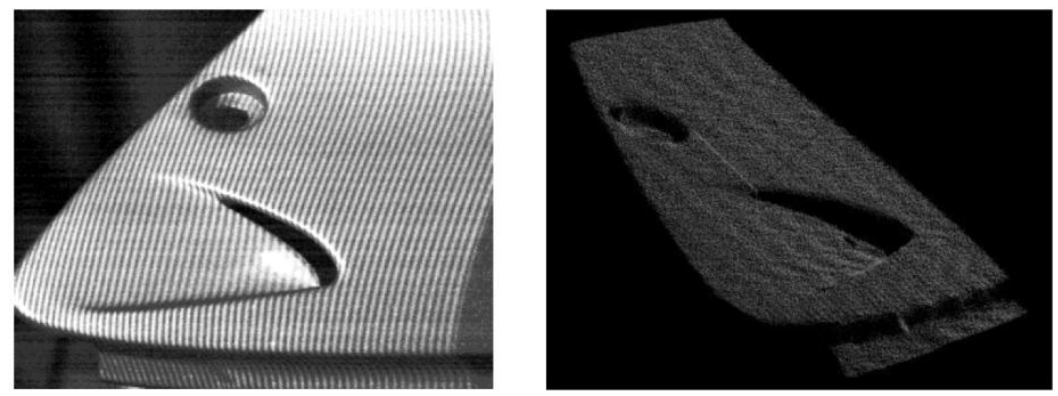

Fig. 20. Motorcycle protection plate testing.
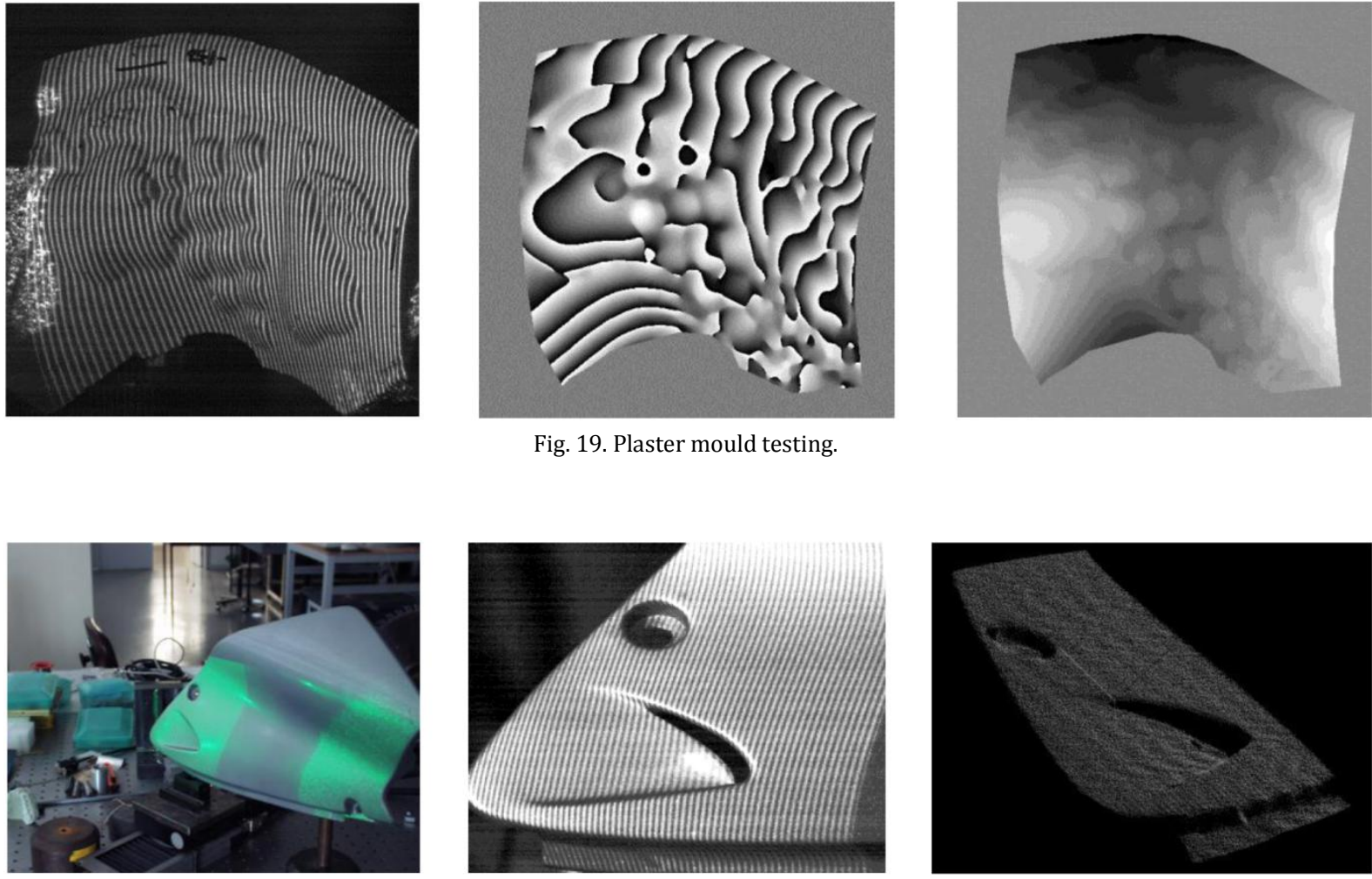

\section{A simple technique for correcting image distortion in fringe projection methods}

\section{6.a. Introduction}

It is not uncommon to observe imaging distortion artifacts in full-field fringe projection systems, due to the system's inability to reproduce the ideal sinusoidal patterns projected onto the object. This is a particularly stringent problem with multi-frequency methods such as temporal phase unwrapping (TPU), because inadequate width results in wrong or incomplete fringe superposition, necessary to the phase-shift algorithm. A less visible effect, but one which can still degrade final image quality, is the contrast degradation imposed by the system's impulse response width: the response of an incoherent system to a single frequency sinusoidal pattern degrades the object contrast or modulation by the complex optical transfer function (OTF) amplitude, thus called modulation transfer function (MTF).
An example of such a 3D surface acquired with the temporal phase unwrapping technique can be seen in Fig. 21.

There are several causes to image degradation, although it seems the limiting factor is the reduced contrast ratio of digital light processors (DLP) or liquid crystal displays (LCD) currently being used as light modulators. In particular, the high black level observed with these projectors, due to the fact that either the crossed polarisers in LCD displays or the partial "ON" duty cycle, even in no signal black scenes in DLPs, results in some unintended light always being transmitted to the scene. This is exemplified in Fig. 21, which shows the effect of a typical projector device on an ideal sinusoidal pattern and the consequences on the final acquired 3D surface. A simple solution to this problem is suggested by the OTF analysis, and has been successfully applied to the TPU method in our lab. We basically seek the periodic function which has to be projected so the final image is a near perfect sinusoid. 


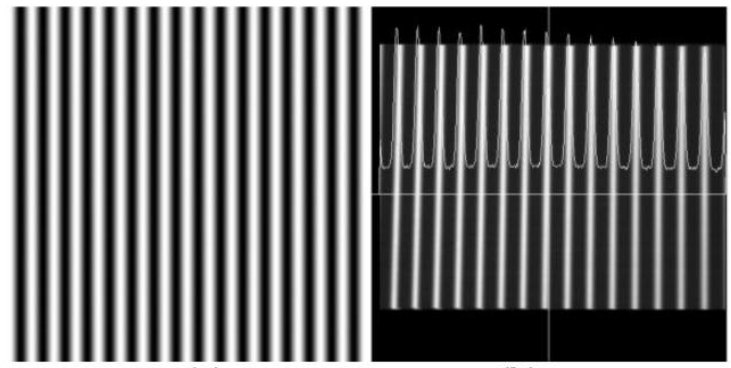

(a)

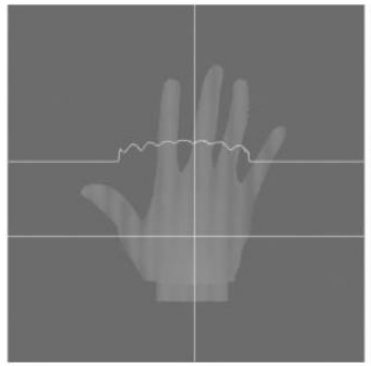

(c) (b)

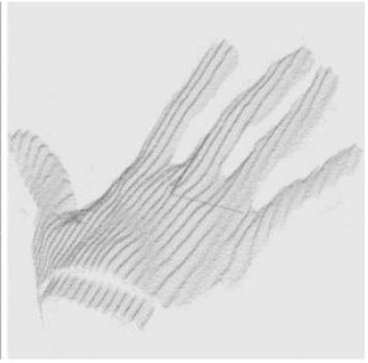

(d)

Fig. 21. A simple surface acquired with TPU. (a) a 16 fringes sinusoid; (b) the real image as seen through the imaging system; (c) a point cloud obtained with the 16 fringe patterns, where a line profile shows the acquisition error due to the imaging distortions; (d) the 3D representation of the acquired surface.

\section{6.b. Analysis}

In order to find the image necessary for each projected frequency, we start by calculating the complex OTF for the projection and imaging system, henceforth called imaging system.

The OTF of an incoherent imaging system can be defined as the system's ability to transfer contrast from object to image. It is the Fourier transform of the system's impulse response, i.e., the imaging of a single point source, which can be obtained by using the Huygens-Fresnel integral to follow the propagation of light from the point source through the system [29,30]. Incoherent imaging systems can be considered linear in intensity. This means that

$$
\begin{aligned}
& I_{i}(x, y)=I_{o}(x, y) \otimes I R(x, y)= \\
& =\int_{-\infty}^{+\infty} \int_{o}(u, v) I R(x-u, y-v) d u d v \Rightarrow \\
& \quad \Rightarrow F\left(I_{i}(x, y)\right)=F\left(I_{o}(x, y)\right) \operatorname{OTF}(u, v),
\end{aligned}
$$

where $I_{i}$ is the image intensity, $I_{o}$ is the object intensity, $I R$ is the system's impulse response and $\otimes$ is the usual convolution operator. In

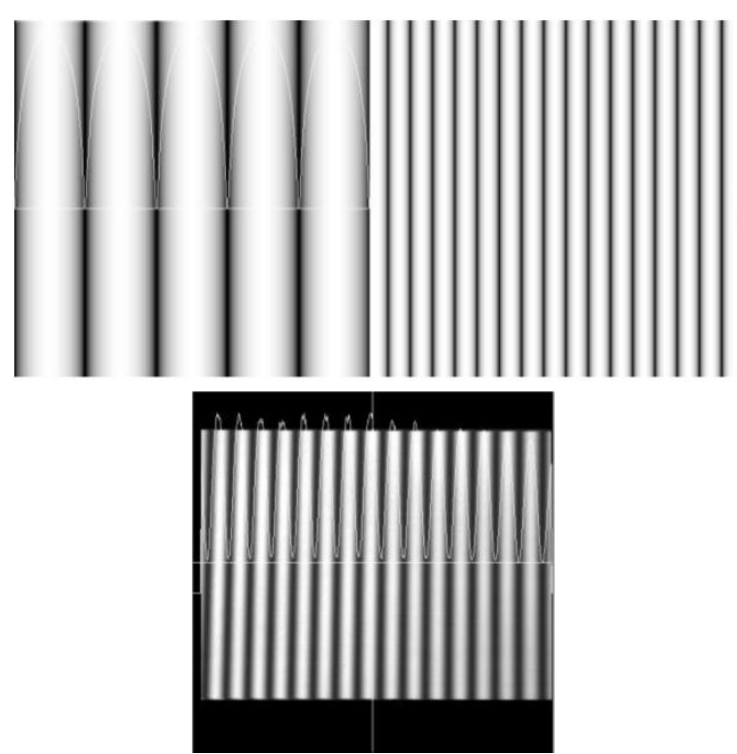

Fig. 22. Artificial patterns to obtain sinusoidal fringes when sent through the imaging system.

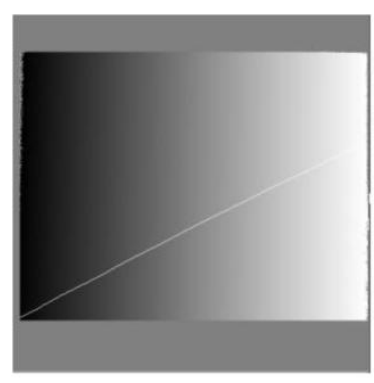

(a)

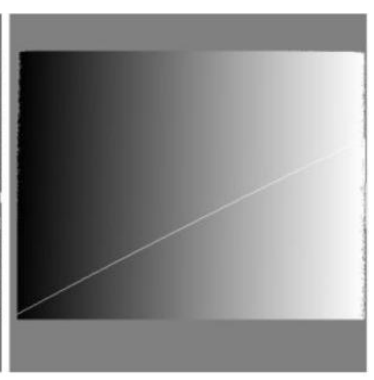

(b)

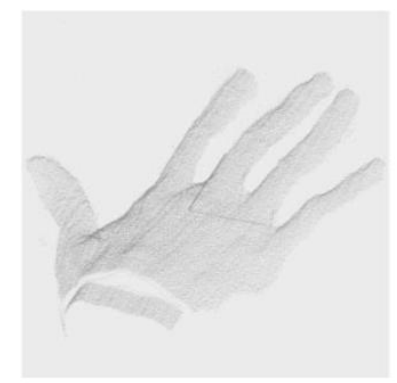

(c)

Fig. 23. A reference plane captured with 5 and 16 fringes and; (c) the surface from Fig. 21 with 16 fringes.

reciprocal space, the use of the convolution theorem implies the Fourier transform of the image intensity is equal to the product of the Fourier transform of the object intensity and the Fourier transform of the impulse response, which is the OTF of the imaging system.

This provides a simple way to calculate the complex OTF of the imaging system. Now, for 
each ideal sinusoid pattern, there must be some artificially distorted image which, when sent to the projector and imaged through the system, will result in a perfect sinusoid, i.e.,

$$
\begin{gathered}
I_{\text {ideal }}(x, y)=I_{?}(x, y) \otimes I R(x, y) \Rightarrow \\
\Rightarrow F\left(I_{?}(x, y)\right)=\frac{F\left(I_{o}(x, y)\right)}{\operatorname{OTF}(u, v)} \Rightarrow \\
\Rightarrow I_{?}(x, y)=F^{-1}\left\{\frac{F\left(I_{o}(x, y)\right)}{\operatorname{OTF}(u, v)}\right\},
\end{gathered}
$$

which is a straightforward way to calculate this artificial image.

We have used an InFocus LP70R DLP projector and a Kodak ES1.0 camera for testing. The artificial images have been created as described above and saved for later projection. As shown in Fig. 22 for 5 and 16 fringes, these images are much narrower on the black level, as expected, which is bound to compensate for the limited contrast ratio of the projector.

The effect on a reference plane and on the previously captured surface can be seen in Fig. 23.

\section{6.c. Conclusion}

The performance of imaging and projection systems can seriously hinder the quality of fullfield fringe projection methods for 3D surface acquisition or displacement measurements. A solution to the poor contrast ratio exhibited by these systems was proposed and tested with satisfactory results.

\section{Acknowledgements}

Acknowledgments are in order to the entire crew at LOME, namely Prof. Mário Vaz, Eng. Nuno Ramos, Dr. Jaime Monteiro and Eng. Jorge Reis. The work described in this note was entirely supported by LOME and INEGI - The Portuguese Mechanical Engineering and Industrial Management Institute. 